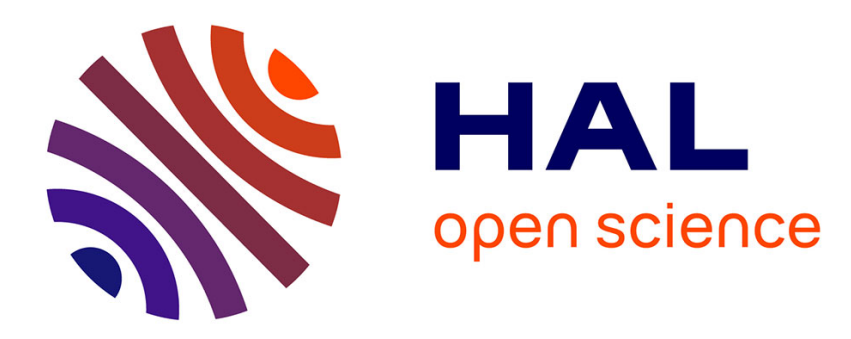

\title{
Normal stress differences in non-Brownian fiber suspensions
}

S Bounoua, P Kuzhir, E Lemaire

\section{To cite this version:}

S Bounoua, P Kuzhir, E Lemaire. Normal stress differences in non-Brownian fiber suspensions. Journal of Rheology, 2016, 60 (4), pp.661. 10.1122/1.4954084 . hal-01356269

\section{HAL Id: hal-01356269 \\ https://hal.science/hal-01356269}

Submitted on 25 Aug 2016

HAL is a multi-disciplinary open access archive for the deposit and dissemination of scientific research documents, whether they are published or not. The documents may come from teaching and research institutions in France or abroad, or from public or private research centers.
L'archive ouverte pluridisciplinaire HAL, est destinée au dépôt et à la diffusion de documents scientifiques de niveau recherche, publiés ou non, émanant des établissements d'enseignement et de recherche français ou étrangers, des laboratoires publics ou privés. 
AIP/123-QED

\title{
Normal stress differences in Non-Brownian fiber suspensions
}

\author{
S. Bounoua, P. Kuzhir, and E. Lemaire* \\ LPMC UMR 7336 - CNRS Université de Nice Sophia-Antipolis \\ Parc Valrose - 06108 Nice cedex 2 - France
}

(Dated: June 19, 2016)

\begin{abstract}
In this paper, we present an experimental study of the normal stress differences that arise in nonBrownian rigid fiber suspensions subject to a shear flow. While early measurements of the normal stress in fiber suspensions in Newtonian fluids measured only $N_{1}-N_{2}$, the recent work of Snook et al. J. Fluid Mech. 758486 (2014) and the present paper provide the first measurements of $N_{1}$ and $N_{2}$ separately. Snook et al perform such measurements with a gap that is very wide compared with the fiber length, whereas the present paper explores the effects of confinement when the gap is 4-10 times the fiber length. The first and the second normal stress differences are measured using a single experiment which consists of determining the radial profile of the second normal stress, along the velocity gradient direction, $\Sigma_{22}$, in a torsional flow between two parallel discs. Suspensions are made of monodisperse fibers immersed in a neutrally buoyant Newtonian fluid. Two fiber lengths and three aspect ratios $a_{r}=L / d$, and a wide range of concentrations have been tested. $N_{1}$ is found to be positive while $N_{2}$ is negative and the magnitude of both normal stress differences increases when $n L^{2} d$ increases, $\mathrm{n}$ being the number fraction of fibers. The magnitude of $N_{2}$ is found to be much smaller than $N_{1}$ only for high aspect ratios and low fiber concentrations. Otherwise, $N_{1}$ and $N_{2}$ are of the same order of magnitude. This is in contradiction with what is often assumed (i.e. $\left|N_{2}\right|<<N_{1}$ ) but consistent with the recent numerical work of Snook et al. that includes contact interactions. The effect of confinement on $N_{1}$ and $N_{2}$ is studied and it is shown that the more confined the suspension, the greater the magnitude of the normal stress differences. At last, the surface properties of the fibers are changed and the impact on the normal stress differences is discussed.
\end{abstract}

* elemaire@unice.fr 


\section{INTRODUCTION}

Fiber suspension rheology is involved in many industrial processes that make use of the reinforcement of polymeric materials with short fibers. The question of fiber suspension rheology is rather complicated since there is a strong coupling between the flow, the fiber orientation and the rheological properties. Most theoretical approaches have considered purely hydrodynamic interactions between particles [? ? ? ? ] that, in non-Brownian fiber suspensions, would give rise to zero or weak normal stress differences [? ] thanks to reversibility of the Stokes flow implying a symmetry of the fiber orientation distribution with respect to the velocity gradient. Particle level simulations [? ? ? ] have shown that direct mechanical contacts and Coulombic friction forces between fibers still give a linear scaling of the suspension stress on the shear rate but perturb the symmetry of the orientation distribution, thus leading to non-zero normal stress differences. The first normal stress difference $N_{1}$ has been found to be positive, while the second one, $N_{2}$, is negative and its absolute value is usually much lower than that of the first normal stress difference, at least for fibers of high aspect ratio. Some semi-phenomenological theories have also been developed to account for direct contacts between fibers by adding a diffusion term to the Jeffery equation [? ] and have been able to correctly predict fiber orientation distribution in the semi-dilute regime with an asymmetry induced by fiber-fiber collisions. In the weak diffusion limit, such an approach predicts the first normal stress difference scales with $a_{r}^{4} / \ln \left(a_{r}\right)$, where $a_{r}$ is the fiber aspect ratio. However the experimental data from different authors (analyzed in details in the paper of Zirnsak et al. [? ] scale much better with the prediction of Carter [? ]: $N_{1} \sim a_{r}^{3 / 2} / \ln \left(a_{r}\right)$. Some authors [? ? ? ] have developed an analytical model including short-range hydrodynamic interactions between fibers and their collisioninduced diffusion. They obtained both transient and steady-state responses for the difference $N_{1}-N_{2}$. The model results fits at least semi-quantitatively to the measurements in parallel plate geometry. However, the relative importance of the second normal stress difference has not been reported. Besides mechanical contacts, fiber flexibility can also lead to nonzero normal stress differences in non-colloidal fiber suspensions, as shown theoretically by Keshtkar et al. [? ] and by particle level simulations [? ? ].

Besides these conceptual difficulties, experimental measurements of the normal stress

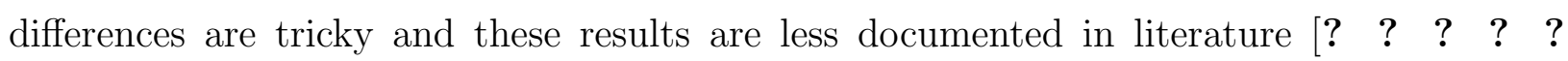


] than the measurements of shear viscosity. Actually, in most studies [? ? ? ], it is the difference between the first and the second normal stress differences, $N_{1}-N_{2}$, that is measured. Indeed, usually a parallel plate geometry is used and $N_{1}-N_{2}$ is directly determined from the measurement of the net thrust force exerted on one of the disks. Then, $N_{2}$ is commonly assumed to be much smaller than $N_{1}[? ?$ ? , so that the measurement of the thrust force is expected to give an estimate of $N_{1}$. Nevertheless, recently, Snook et al. [? ] have measured for the first time $N_{1}$ and $N_{2}$ independently, or more precisely the two normal stress coefficients, $\alpha_{1}$ and $\alpha_{2}$ that are the ratios of $N_{1}$ and $N_{2}$ to the shear stress. The values of $\alpha_{1}$ and $\alpha_{2}$ measured for aspect ratios comprised between 12 and 32 are deduced from the deformation of the free surface of fiber suspensions flowing in a tilted trough and in a Weissenberg rheometer and have shown that the magnitude of the first normal stress difference, which is positive, is only approximately twice that of the second normal stress difference which is negative. These measurements were performed with a gap that is very large compared to the fiber length whereas the present paper explores the effects of confinement when the gap is 4-10 times the fiber length.

The present paper is indeed devoted to the measurement of the first and the second normal stress differences in fiber suspensions using an alternative method based on a device previously designed by Dbouk et al. [? ? ]. This device has been already used to measure both $N_{1}$ and $N_{2}$ in concentrated suspensions of spherical particles. Note that, in contrast to what had been obtained before for spherical particles [? ? ? ], Dbouk et al. [? ] measured a positive first normal stress difference while, as in the previous studies, $N_{2}$ was found to be negative. Recently, the same qualitative behaviour has been reported by Gamonpilas et al. [? ] (i.e. $N_{1} \gtrsim 0$ and $N_{2}<0$ ) and the authors have shown that the sign of the first normal stress difference was very sensitive to the polydispersity of the spherical particles. In particular they compare the values of $N_{1}$ and $N_{2}$ obtained for two monodisperse and two bidisperse non-Brownian suspensions for two particle concentrations, 0.4 and 0.5. $N_{1}$ is found almost equal to zero for the bidisperse suspensions while it is positive for the monodisperse suspensions even though much smaller than the values obtained by Dbouk et al.

In the present paper, the measurements are carried out for neutrally buoyant suspensions made of short rigid fibers dispersed in a Newtonian fluid for 2 fiber lengths, L, 3 aspect ratios, $a_{r}=L / d$, where $\mathrm{d}$ is the fiber diameter and for volume concentrations ranging from 0.03 to 
0.25. The measurement principle is described in Sec. ?? and details on fiber suspensions, experimental device and measurement procedure are given in Sec. ??. Results are presented and discussed in the last section of the paper where the fiber concentration dependence of $N_{1}$ and $N_{2}$ is presented as well as the impact of the confinement and of the fiber surface properties on the normal stress differences.

\section{DETERMINATION OF THE MATERIAL FUNCTIONS IN PARALLEL-PLATE GEOMETRY}

\section{A. Material functions}

We consider a suspension of rigid rod-like particles immersed in a Newtonian fluid. The viscosity of the suspension, $\eta_{s}$, depends on the fiber volume fraction, $\phi$ and on the fiber aspect ratio, $a_{r}$. The suspension is subjected to a shear stress $\Sigma_{12}$ in a torsional flow between two rotating parallel discs, the indices 1 and 2 denoting the direction of the shear velocity and of the velocity gradient respectively.

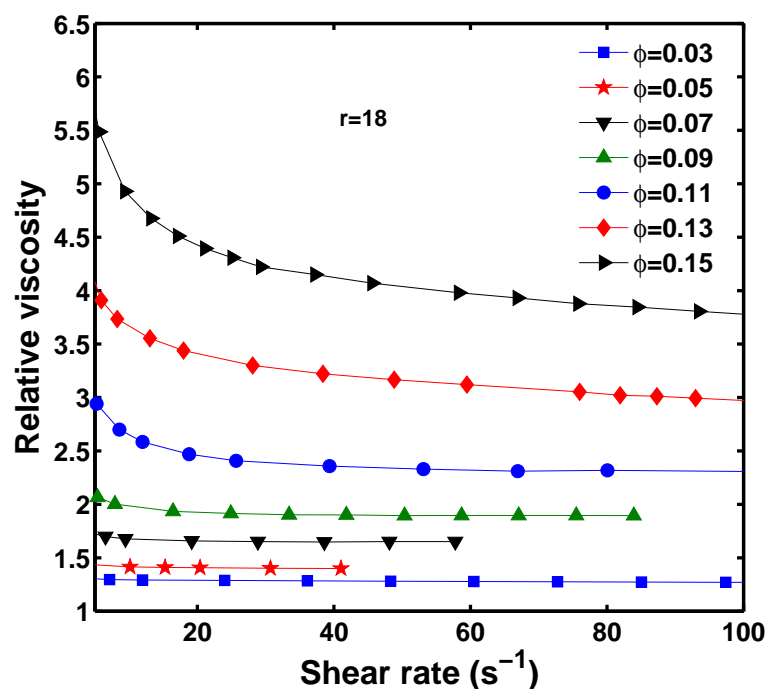

FIG. 1. Relative shear viscosity, $\eta_{r}$, versus shear rate for suspensions of fibers with an aspect ratio $a_{r}=18$ and for different concentrations.

The variation of the relative viscosity $\eta_{r}=\frac{\eta_{s}(\phi)}{\eta_{f}}$, where $\eta_{f}$ is the viscosity of the suspending fluid, with shear rate, $\dot{\gamma}$, is shown in Fig. ??. First, we have to mention that the 
viscosity reported here is actually the apparent viscosity that is the ratio of the shear stress at the rim to the shear rate the rim and not the true viscosity that should have been obtained by deconvoluting the data with various shear stress and rates at the rim. Nevertheless, it clearly appears that fiber suspensions are shear-thinning when the fiber concentration is high enough. The higher the fiber concentration and aspect ratio, the more pronounced is the shear-thinning behaviour (see, for instance, [? ]). Such a shear thinning behaviour has often been reported [? ? ? ? ? ] whereas its origin has not received any clear explanation. Most models either theoretical [? ? ] or numerical [? ? ? ] do not report such a shear-thinning behaviour because the effect of short-range hydrodynamic forces and of direct mechanical contacts that are all supposed to be proportional to the shear rate, leading to a linear scaling of the shear stress with the shear rate. Shear thinning can only occur if a characteristic time different from $1 / \dot{\gamma}$ is involved in the dynamics of the suspension. In particular, this is the case if the contacts are adhesive or if the friction is not Coulombic (i.e. a non-linear frictional law). The ratio of the characteristic adhesive force $\mathrm{F}$ to the characteristic hydrodynamic force can be evaluated [?]:

$$
N \approx \frac{F}{\eta_{f} \dot{\gamma} d^{2}}
$$

If $N>1$, adhesive forces are dominant and are expected to play a role in the suspension rheology while they can be neglected if $N<1$. We deduce the critical shear rate value that has to be applied for the suspension to behave as a viscous liquid:

$$
\dot{\gamma}_{c} \approx \frac{F}{\eta_{f} d^{2}}
$$

An order of magnitude for the adhesive force is given by Chaouche et al. [? ]: $F \approx 10^{-9}-10^{-8} N$ for polyamide fibers with size close to that of the fibers used in the present experiments. Thus, according to the values of the suspending liquid viscosity and of the fiber diameter, we obtain the following estimate for the critical shear rate above which there is no or slight shear thinning: $\dot{\gamma}_{c} \approx 10-100 \mathrm{~s}^{-1}$. This order of magnitude is consistent with the measurements presented in Fig. ??.

In the following, the normal stress differences will be measured in the shear rate range of approximately 20 to $50 \mathrm{~s}^{-1}$ and the residual shear-thinning will be described by a power law:

$$
\Sigma_{12}=K(\phi) \dot{\gamma}^{n}
$$


where $n \leq 1$ is the shear-thinning index and $K(\phi)$, the consistency.

When the fiber concentration is high enough, anisotropic normal stresses, $\Sigma_{11}, \Sigma_{22}$ and $\Sigma_{33}$ arise [? ? ? ]. Then, two important quantities are introduced: the first and the second normal stress differences, defined as :

$$
N_{1}(\dot{\gamma})=\Sigma_{11}-\Sigma_{22} \quad, \quad N_{2}(\dot{\gamma})=\Sigma_{22}-\Sigma_{33}
$$

where the index 3 denotes the direction of the vorticity. Normal stress differences in fiber suspensions are known to scale linearly with the shear stress [? ? ], even for shear thinning suspensions, as can be observed in [? ]. Hence, we introduce the first and the second normal stress coefficients $\alpha_{1}(\phi)$ and $\alpha_{2}(\phi)$ :

$$
N_{1}(\dot{\gamma})=\alpha_{1}(\phi) \Sigma_{12} \quad, \quad N_{2}(\dot{\gamma})=\alpha_{2}(\phi) \Sigma_{12}
$$

The linear scaling of $N_{1}$ and $N_{2}$ with the shear stress will be rediscussed in Sec. ??.

\section{B. Determination of the normal stress differences}

Here we present the method to determine the values of the first and the second normal stress differences. $N_{1}$ and $N_{2}$ are deduced from the radial profile of the normal stress along the velocity gradient direction, $\Sigma_{22}$, in a torsional flow between two parallel discs separated by a distance $h$. The flow is generated by a torque, $\Gamma$, applied to the upper disc that rotates with an angular velocity, $\Omega$. The shear rate $\dot{\gamma}(r)=\frac{\partial v_{\theta}}{\partial z}$ depends linearly on $r$. In this geometry, the components of the suspension velocity are:

$$
v_{r}=0, \quad v_{\theta}(r)=r \Omega(z), \quad v_{z}=0
$$

and the shear rate is:

$$
\dot{\gamma}(r)=\frac{\Omega r}{h}
$$

Starting from the equation of motion for Stokes flow in cylindrical coordinates [? ]:

$$
\begin{array}{cl}
r \text {-component } & \frac{\partial \Sigma_{33}}{\partial r}+\frac{\Sigma_{33}-\Sigma_{11}}{r}=0 \\
\theta-\text { component } & \frac{\partial \Sigma_{12}}{\partial z}=0
\end{array}
$$


and expressing $\Sigma_{11}$ and $\Sigma_{33}$ in terms of $N_{1}, N_{2}$ and $\Sigma_{22}$ and using Eq.(??), we obtain the radial profile of the second normal stress $\Sigma_{22}$ :

$$
\frac{\partial \Sigma_{22}}{\partial r / R}=\Sigma_{12}(R)\left(\alpha_{1}+(n+1) \alpha_{2}\left(\frac{r}{R}\right)^{n-1}\right)
$$

where $\Sigma_{12}(R)$ is the shear stress at the rim. After integrating equation (??) and assuming that at the air/suspension interface, $\Sigma_{33}(R)$ is the sum of capillary and atmospheric pressure:

$$
\Sigma_{33}(r=R)=P_{C a p}+P_{a t m}=P_{r e f}
$$

We obtain the expression of $\Sigma_{22}$ :

$$
\begin{aligned}
& \Sigma_{22}(r / R)=\Sigma_{12}(R)\left(\frac{\alpha_{1}+(n+1) \alpha_{2}}{n}\left(\frac{r}{R}\right)^{n}-\frac{\alpha_{1}+\alpha_{2}}{n}\right) \\
& +P_{r e f}
\end{aligned}
$$

where $p_{\text {ref }}=p_{\text {cap }}+p_{\text {atm }}$ is the reference pressure that is, as verified in [? ], independent of the shear rate.

Eq. (??) shows that the radial profile of the second normal stress $\Sigma_{22}$ is expected to be proportional to the shear stress at the rim and to vary linearly when $\Sigma_{22}$ is plotted against $(r / R)^{n}$. Then, from a linear combination of the slope, $A$, and the ordinate at the origin, $B$, the values of the two normal stress coefficients $\alpha_{1}(\phi)$ and $\alpha_{2}(\phi)$ can be deduced.

$$
\left\{\begin{array}{l}
\alpha_{1}(\phi)=-A-(1+n) B \\
\alpha_{2}(\phi)=A+B
\end{array}\right.
$$

Note that for a non shear-thinning suspension $\left(\Sigma_{12} \propto \dot{\gamma}, \mathrm{n}=1\right)$,Eq. (??) reduces to the formula previously reported by Dbouk et al. [? ]:

$$
\Sigma_{22}(r / R)=\Sigma_{12}(R)\left(\left(\alpha_{1}+2 \alpha_{2}\right) \frac{r}{R}-\left(\alpha_{1}+\alpha_{2}\right)\right)+P_{r e f}
$$

\section{EXPERIMENT}

\section{A. Fiber suspensions}

\section{Suspending liquid}

The suspending liquid is a Newtonian fluid made of a mixture of pure water, Zinc Bromide (Sigma-Aldrich, France) and Ucon oil 75H90000 (Dow Chemical, France; density 1.09 
$\mathrm{g} / \mathrm{cm}^{3}$, viscosity 30 Pas at $\left.25^{\circ} \mathrm{C}\right)$. According to the ratio of these three components in the mixture, both its density and its viscosity can be controlled. The viscosity of the suspending liquid is tuned by changing the fraction of Ucon oil in such a way that the viscosity of the whole suspension is of the order of 1 Pa.s, which, as explained in Sec. ??, allowed us to measure normal stress differences under the best conditions. To achieve this whatever the fiber concentration, the relative proportions of water and Ucon oil are varied in order to obtain the desired suspending liquid viscosity that can vary from 0.1 to 1 Pa.s. (see Table ?? in Sec. ??). An appropriate amount of zinc Bromide is added into the mixture in order to adjust the density of the suspending liquid to that of the fibers which is either 1.09 or $1.34 \mathrm{~g} / \mathrm{cm}^{3}$ (see Table ??). Note that the viscosity of the suspending liquid does not change with the shear rate in the studied shear rate range and that, as a consequence, the shear thinning of the fiber suspensions reported in Fig. 1 does not come from the non-Newtonian behavior of the solvent but rather from non-hydrodynamic interactions between fibers.

\section{Fibers and concentration regimes}

Four batches of monodispersed polyamide fibers provided by Société Nouvelle Le Flockage were used in the experiments with three aspect ratios, $a_{r}=10,18$ and 33, and two lengths, $\mathrm{L}=300$ and $500 \mu \mathrm{m}$. The fibers are quite monodispersed. Their size distribution has been measured by optical microscopy and has been found to be of the order of $\pm 5 \mu \mathrm{m}$ for the length and lower than $\pm 1 \mu m$ for the diameter, $\pm 1 \mu m$ corresponding to our optical measurement resolution. This estimation of the polydispersity has been conducted on about 80 fibers for each batch.

A series of fiber volume fractions was prepared in order to explore both the semi dilute regime $\left(n L^{3}>1, n L^{2} d<1\right)$ and the concentrated regime $\left(n L^{2} d>1\right)[?]$. In these two regimes, fibers are expected to interact through both hydrodynamic and contact forces [? ? ]. The fiber volume concentrations ranged from 0.03 to 0.25 leading to concentration parameter, $n L^{2}$, comprised between 1 and 6 , depending on fiber aspect ratio.

Fibers can be considered as rigid since the shear stress is much smaller than the critical stress $\Sigma_{\text {crit }}$ at which the fibers may be expected to buckle under shear compression. $\Sigma_{\text {crit }}$ is 
given by [? ]:

$$
\Sigma_{c r i t} \cong \frac{E_{b}\left(\ln 2 a_{r}-1.75\right)}{2 a_{r}^{4}}
$$

$E_{b}$ is the bending modulus of the fibers of the order of $3.10^{9} \mathrm{~Pa}$ for polyamide. Table ?? summarizes the characteristics of the fibers (length, diameter, density) and gives the values of the fiber volume fraction that separate the different concentration regimes and the ratio of the critical buckling stress to the maximum applied shear stress, $\Sigma_{\max }$.

\begin{tabular}{ccccccc}
\hline \hline Aspect ratio Length Diameter density Concentration regime limits & $\frac{\Sigma_{\text {crit }}}{\Sigma_{\text {max }}}$ \\
& $L(\mu)$ & $\mathrm{d}(\mu \mathrm{m})$ & $g / \mathrm{cm}^{3}$ & $\phi^{\prime}=\pi /\left(4 a_{r}^{2}\right)$ & $\phi^{\prime \prime}=\pi /\left(4 a_{r}\right)$ \\
\hline 18 & $500 \pm 5$ & $28 \pm 1$ & 1.09 & 0.0024 & 0.044 & 440 \\
18 & $300 \pm 5$ & $17 \pm 1$ & 1.09 & 0.0024 & 0.044 & 220 \\
10 & $300 \pm 5$ & $30 \pm 1$ & 1.09 & 0.0079 & 0.079 & 1400 \\
33 & $500 \pm 5$ & $15 \pm 1$ & 1.34 & $7.210^{-4}$ & 0.024 & 22 \\
\hline
\end{tabular}

TABLE I. Characteristics of fiber suspensions studied in our experiments. $\phi^{\prime}=\pi /\left(4 a_{r}^{2}\right)$ and $\phi^{\prime \prime}=\pi /\left(4 a_{r}\right)$ denote the characteristic volume fractions that separate the dilute regime from the semi dilute and the semi-dilute regime from the concentrated one, respectively. $\Sigma_{\text {crit }}$ is the critical shear stress at which fibers are expected to buckle and $\Sigma_{\text {max }}$, the maximum applied shear stress in our experiment.

\section{Preparation of the suspensions}

Before preparing the suspensions, fibers are washed out with distilled water and put in an ultrasonic bath for a few minutes in order to eliminate the coating on their surface. The fibers are then placed in an oven at $35^{\circ} \mathrm{C}$ for 12 hours in order to be dried. This washing procedure was applied to all suspensions except those whose rheology is described in Appendix A.

Then, fibers are added to the suspending liquid and the suspension is gently mixed with a spatula. The suspension is then placed in a centrifuge for a few tens of minutes and in an ultrasonic bath for a period of 2 to 4 hours, in order to remove air bubbles. After that, they 
are again gently mixed in order to resuspend, if necessary, the particles without including air bubbles.

\section{B. Measurements}

1. Experimental device

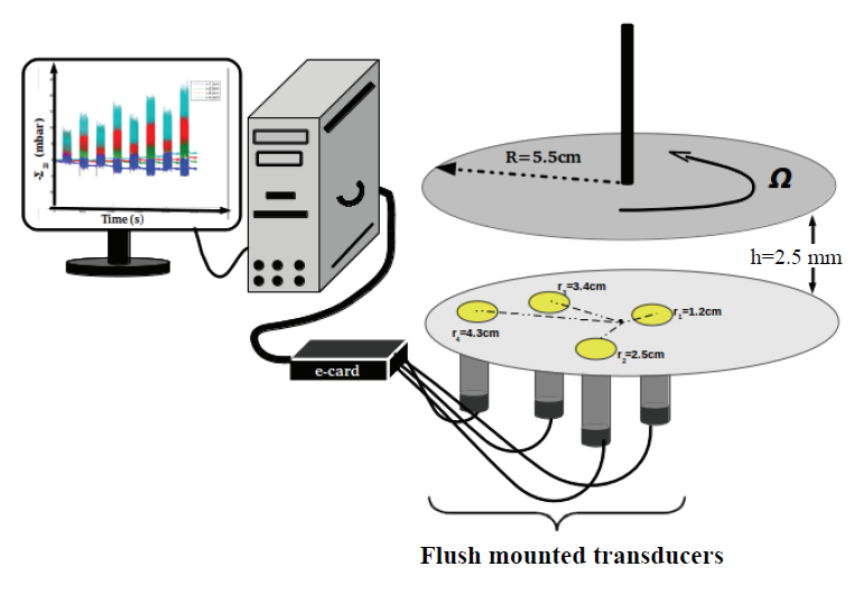

FIG. 2. Experimental device used to measure the radial profile of $\Sigma_{22}$. The lower disc is equipped with four flush pressure transducers placed at different radial positions: $\left(r_{1}=1.2 \mathrm{~cm}, r_{2}=2.5 \mathrm{~cm}\right.$, $r_{3}=3.4 \mathrm{~cm}$ and $\left.r_{4}=4.3 \mathrm{~cm}\right)$.

The normal stress difference measurements are conducted using a controlled-stress rheometer HAAKE MARS II in parallel plate geometry. The suspension is placed between two parallel discs of radius $R=5.5 \mathrm{~cm}$ separated by a $h=2.5 \mathrm{~mm}$ gap. The lower disc is equipped with four flush mounted differential pressure transducers $(A T M \pm 25$ mbar, STS ) and placed at different radial positions as shown in Fig. ??. These pressure transducers allow us to measure the radial profile of $\Sigma_{22}$ and then the two normal stress coefficients, $\alpha_{1}$ and $\alpha_{2}$, as explained in Sec. ??. The transducers have to be placed right at the surface of the lower disc in order to avoid hole pressure effects that would lead to extra pressure coming from flow perturbation. To this purpose, all transducers are placed slightly below the level of the surface of the lower disc (about 50 to $100 \mu \mathrm{m}$ ) and topped with a paraffin layer. The transducer responses are tested by measuring the hydrostatic 
pressure of a liquid column and also, as detailed in [? ], the inertial pressure generated by the torsionnal flow of a Newtonian liquid, even at low Reynolds number.

\section{Flow regime}

In our experiment, we consider only the case of laminar flow and, for each studied suspension, we check that for the highest angular velocity, $\Omega$, the Reynolds number, $N_{R e}$ remains small:

$$
N_{R e}=\frac{\rho \Omega R h}{\eta_{s}} \lesssim 0.1
$$

Furthermore, fibers are large enough for hydrodynamic forces to dominate Brownian forces as it is determined by the Péclet Number, $N_{P e}$, that is very large [? ]:

$$
N_{P e}=\frac{\eta_{0} \dot{\gamma} \pi L^{3}}{3 k_{B} T \ln \left(a_{r}\right)} \sim 10^{9} \gg 1
$$

3. Experimental procedure

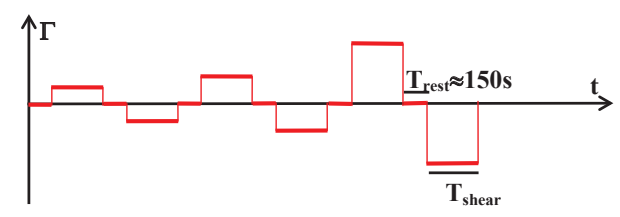

FIG. 3. A series of torques is applied during the same period $T_{\text {shear }}$ in the clockwise and counterclockwise directions. The suspension was left at rest during a time interval, $T_{\text {rest }}$, between rotations in the two opposite directions.

To begin our experiment, we carefully put the suspension on the lower disc taking care not to trap any air bubble, then we place a drop of the suspension on the upper disc and bring it down slowly toward the lower plate until a gap of $2.45 \mathrm{~mm}$ is reached. When the suspension has completely spread across the gap, the extra suspension is carefully cleaned up and the upper disc is raised by $50 \mu \mathrm{m}$. After that, all the transducers are set to zero $\left(P_{r e f}=0\right.$ in eq. (??)). Regarding the effect of slightly raising the gap width, it was observed that the suspension/air interface was more stable as well as the pressure signals. In addition, 
a slight increase of the gap width guaranties that no suspension remains outside the gap which could eventually lead to fiber migration in this region of low shear rate.

The experimental protocol is shown in Fig. ??. We apply a series of torques $\Gamma$ in the clockwise and counter-clockwise directions with a duration $T_{\text {shear }}$, separated by a rest period $T_{\text {rest }}=150 \mathrm{~s}$. It is necessary to apply such a complicated protocol since, due to the large diameter of the discs, even very slight parallelism defects lead to extra pressures that can be of the same order of magnitude as the pressure we aim to measure. In our experiment, the out of roundness of the rotating disc leads to variation of the gap of about $50 \mu \mathrm{m}$. If not corrected, this gap width variation would generate a pressure error, evaluated by [? ]:

$$
\delta p \simeq \eta \Omega R^{2} \frac{\delta h}{h^{3}}=\Sigma_{12} \frac{\delta h R}{h^{2}} \simeq 0.4 \Sigma_{12}
$$

Fortunately, as discussed in [? ], this error cancels when pressure signal are averaged over one revolution of the upper disc in clockwise and in counter-clockwise directions. That is why it is necessary to apply the torque in both directions. By the way, this requirement to average the pressure signal over long time periods prevent us from measuring transient response of the normal stress differences that would, however, be very interesting!

Since we are doomed to only measure the steady normal stress differences, the torque has to be applied during a period, $T_{\text {shear }}$, long enough for the fibers to achieve a steady orientation state even at the position of the most central transducer. The characteristic orientation time for fibers is expected to be of the order of the Jeffrey period, $T_{J}=\frac{2 \pi}{\dot{\gamma}} \times\left(\frac{1}{a_{r}}+a_{r}\right)[?]$. And, since the shear rate at the most central transducer is equal to $: \dot{\gamma}\left(r_{1}\right)=\frac{r_{1}}{R} \dot{\gamma}_{R}$ with $r_{1}=1.2 \mathrm{~cm}$ and $R=5.5 \mathrm{~cm}$, at this radial position, fibers are expected to reach their steady orientation state after a period equal to $T_{J}\left(r_{1}\right) \simeq 5 \times T_{J}(R)$. Thus, we use the criterion: $T_{\text {shear }}>\frac{10 \pi}{\dot{\gamma}_{R}} \times\left(\frac{1}{a_{r}}+a_{r}\right)$. Then, after this transient period, the pressure signals are recorded and it is checked that both the shear viscosity and the axial normal stress do not change anymore and have reached their stationary values.

\section{Operating conditions}

First, we have to define the shear rate range in which the measurement of the normal stress differences will be performed. According to the criterion on the minimum run time fixed in Sec. ?? to ensure that a stationary orientation of the fibers has been reached, 
the shear rate should be as large as possible. In addition, greater is the shear rate, less pronounced is the shear-thinning, meaning that adhesion forces between fibers are more and more negligible and that our suspensions are really representative of non-colloidal fiber suspensions. Nevertheless, the shear rate cannot be increased too much since the Reynolds number has to remain small as well as the inertial pressure that arises from the secondary radial flow, even at low Reynolds number. The inertial pressure scales as the square of the shear rate [?]:

$$
p_{i}=0.15 \rho h^{2} \dot{\gamma}_{R}^{2}\left(\left(\frac{r_{i}}{R}\right)^{2}-1\right)
$$

Hence, the inertial pressure has to be negligible with respect to $\Sigma_{22}$ that arises from normal stress differences. Considering eq.(??) and $\Sigma_{22} \gtrsim 10 P a$, this leads to: $\dot{\gamma}_{R} \lesssim 60 \mathrm{~s}^{-1}$.

Second, the accuracy of the measurements will depend on the suspensions viscosity. As explained in Sec. ??, the suspending liquid is a mixture of distilled water, Ucon oil and Zinc bromide and according to the relative fractions of water and Ucon oil, its viscosity can be tuned from $10^{-3}$ to about 30 Pa.s. The choice of the viscosity is dictated by a compromise between two contradictory requirements. On one hand, since the normal stress differences are expected to be proportional to the shear stress, the suspending liquid viscosity should be as high as possible. On the other hand, if $N_{2}$ is negative, an instability that manifests itself by an edge fracture of the sheared medium appears when the magnitude of $N_{2}$ is larger than a critical value [?] that is of the order of the capillary pressure: $N_{2_{c}} \approx 5 \gamma / h$ where $\gamma$ is the surface tension of the suspension. Taking for $\gamma$ a typical value of $50 \mathrm{mN} . \mathrm{m}^{-1}$, $N_{2_{c}} \approx 100 P a$. Thus the viscosity of the suspending liquid should not be too large. A rather good compromise is to choose the viscosity of the suspending liquid in such a way that the viscosity of the whole suspension is of the order of 1 Pa.s. This limitation in the maximum applicable shear stress is unfortunate since it implies that, for the highest fiber concentrations, the normal stress measurements have to be carried out in a shear stress range where the suspensions are shear-thinning, which complicates data analysis. For each aspect ratio, fiber length and concentration, Table ?? summarizes the range of the applied shear rates at the rim, the viscosity of the suspending liquid, the shear thinning index, $\mathrm{n}$ and the consistency. The shear thinning index and the consistency have been measured in a shear rate range that extends from the smallest shear rate at the most inner transducer (i.e. about five times lower than the smallest shear rate at the rim) to the largest shear rate at the rim and we checked that, within this range, the shear rate-shear stress relation could 
be adequately represented by a power law.

\section{An example of $\Sigma_{22}$ profile determination}

Fig. ?? shows an example of the recorded pressure signals that, due to parallelism defects, are quite noisy. Despite the poor quality of the pressure signals, axial normal stress $\Sigma_{22}$ is obtained for different values of the applied shear stress, $\Sigma_{12}$ upon averaging the pressure signal, once the steady state is reached, over several revolutions in the clockwise and counterclockwise directions. Fig. ?? shows an example of the radial variation of the measured $\Sigma_{22}$ for $\phi=0.21, a_{r}=10$ and for four different values of the shear stresses. The error bar calculation accounts for the standard error of the recorded signal and of the inaccuracy of the pressure measurement that is evaluated to about $\pm 2.5 \mathrm{~Pa}$.

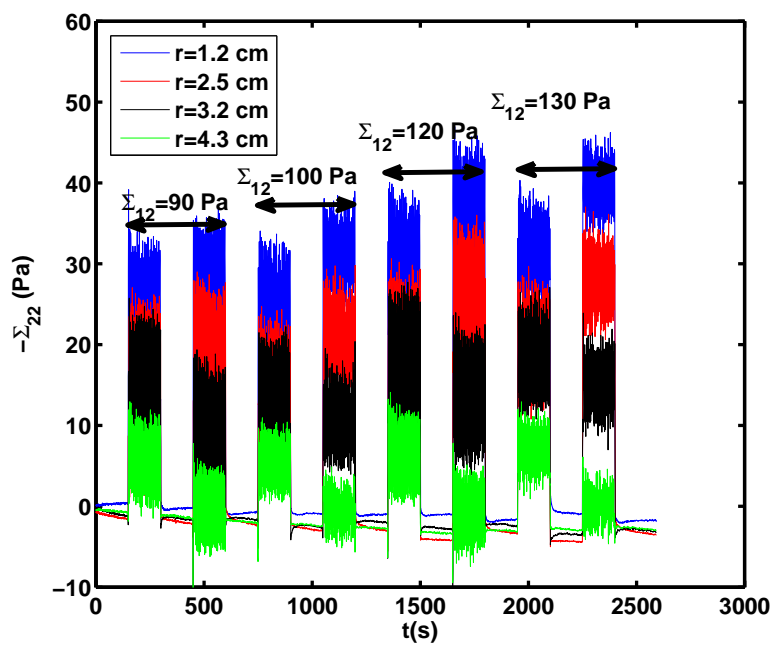

FIG. 4. An example of recorded signals provided by the transducers situated at different radial positions $r_{i}$. This measurement has been performed for a suspension with $a_{r}=10$ and $n L^{2} d=2.7$.

As expected from Eq. (??), when $\Sigma_{22}$ is plotted against $(r / R)^{n}$, the profiles are linear and Fig. ?? indicates that, when $\Sigma_{22}$ is normalized by the shear stress at the rim $\Sigma_{12}(R)$, all profiles collapse onto a single curve. In particular, this collapse, which is obtained whatever the fiber aspect ratio and concentration, means that normal stress are proportional to the shear stress. This result, first shown by Carter [? ] for suspensions which do not shear-thin, 


\begin{tabular}{|c|c|c|c|c|c|c|}
\hline fiber type & $\phi$ & $n L^{2} d$ & $\dot{\gamma}_{R}$ range $\left(s^{-1}\right)$ & $\eta_{f}($ Pas $)$ & $n$ & $K / \eta_{f}\left(s^{n-1}\right)$ \\
\hline & 0.08 & 1.8 & {$[29.3: 44.1]$} & 1.15 & 0.99 & 2.5 \\
\hline & 0.10 & 2.3 & {$[35.1: 44.9]$} & 1.15 & 0.96 & 2.7 \\
\hline & 0.11 & 2.5 & {$[34.2: 45.0]$} & 0.335 & 0.96 & 3.9 \\
\hline$a_{r}=18$ & 0.12 & 2.75 & {$[31.2: 44.7]$} & 0.355 & 0.92 & 4.1 \\
\hline \multirow[t]{6}{*}{$\mathrm{L}=500 \mu$} & 0.13 & 3.0 & {$[25.3: 44.0]$} & 0.355 & 0.93 & 4.7 \\
\hline & 0.14 & 3.2 & {$[29.3: 45.7]$} & 0.355 & 0.90 & 8.1 \\
\hline & 0.15 & 3.4 & {$[27.4: 44.6]$} & 0.173 & 0.87 & 9.3 \\
\hline & 0.10 & 2.3 & {$[23.5: 38.5]$} & 0.324 & 0.95 & 4.0 \\
\hline & 0.11 & 2.5 & {$[21.5: 35.2]$} & 0.324 & 0.94 & 5.36 \\
\hline & 0.12 & 2.75 & {$[15.3: 25.8]$} & 0.324 & 0.90 & 6.9 \\
\hline$a_{r}=18$ & 0.13 & 3.0 & {$[16.3: 35.4]$} & 0.324 & 0.89 & 8.5 \\
\hline \multirow[t]{7}{*}{$\mathrm{L}=300 \mu$} & 0.14 & 3.2 & {$[19.4: 32.4]$} & 0.324 & 0.91 & 10.9 \\
\hline & 0.15 & 3.4 & {$[14.8: 26.3]$} & 0.324 & 0.70 & 29.4 \\
\hline & 0.16 & 3.7 & {$[15.0: 21.7]$} & 0.324 & 0.76 & 37.6 \\
\hline & 0.17 & 3.9 & {$[27.4: 44.6]$} & 0.324 & 0.76 & 41.5 \\
\hline & 0.10 & 1.3 & {$[30.3: 41.3]$} & 1.15 & 0.99 & 2.2 \\
\hline & 0.11 & 1.4 & {$[28.2: 38.2]$} & 1.15 & 0.97 & 2.5 \\
\hline & 0.12 & 1.5 & {$[24.6: 35.1]$} & 1.15 & 0.95 & 3.4 \\
\hline \multirow{11}{*}{$\begin{array}{c}a_{r}=10 \\
\mathrm{~L}=300 \mu\end{array}$} & 0.13 & 1.65 & {$[24.9: 36.2]$} & 1.15 & 0.97 & 3.7 \\
\hline & 0.15 & 1.9 & {$[27.1: 38.7]$} & 0.334 & 0.95 & 4.8 \\
\hline & 0.17 & 2.2 & {$[32.8: 41.5]$} & 0.334 & 0.93 & 7 \\
\hline & 0.19 & 2.4 & {$[35.7: 42.7]$} & 0.334 & 0.90 & 10.7 \\
\hline & 0.20 & 2.5 & {$[17.9: 30.9]$} & 0.164 & 0.86 & 15.2 \\
\hline & 0.21 & 2.7 & {$[26.2: 40.5]$} & 0.334 & 0.89 & 17.8 \\
\hline & 0.23 & 2.9 & {$[16.1: 25.8]$} & 0.324 & 0.73 & 36.5 \\
\hline & 0.25 & 3.2 & {$[25.9: 43.4]$} & 0.324 & 0.61 & 71.6 \\
\hline & 0.03 & 1.3 & {$[31.8: 40.7]$} & 1.067 & 0.99 & 1.8 \\
\hline & 0.05 & 2.1 & {$[27.9: 43.7]$} & 1.067 & 0.99 & 2.1 \\
\hline & 0.07 & 2.9 & {$[25.1: 33.3]$} & 1.067 & 0.95 & 2.2 \\
\hline \multirow{5}{*}{$\begin{array}{c}a_{r}=33 \\
\mathrm{~L}=500 \mu\end{array}$} & 0.085 & 3.6 & {$[23.6: 39.4]$} & 1.067 & 0.99 & 2.8 \\
\hline & 0.10 & 4.2 & {$[33.2: 4 \Phi 5]$} & 0.282 & 0.87 & 12.9 \\
\hline & 0.11 & 4.6 & {$[19.3: 39.4]$} & 0.282 & 0.82 & 13.1 \\
\hline & 0.12 & 5.0 & {$[25.8: 39.8]$} & 0.282 & 0.75 & 19.4 \\
\hline & 0.13 & 5.5 & {$[42.9: 61.5]$} & 0.282 & 0.7 & 30.4 \\
\hline
\end{tabular}




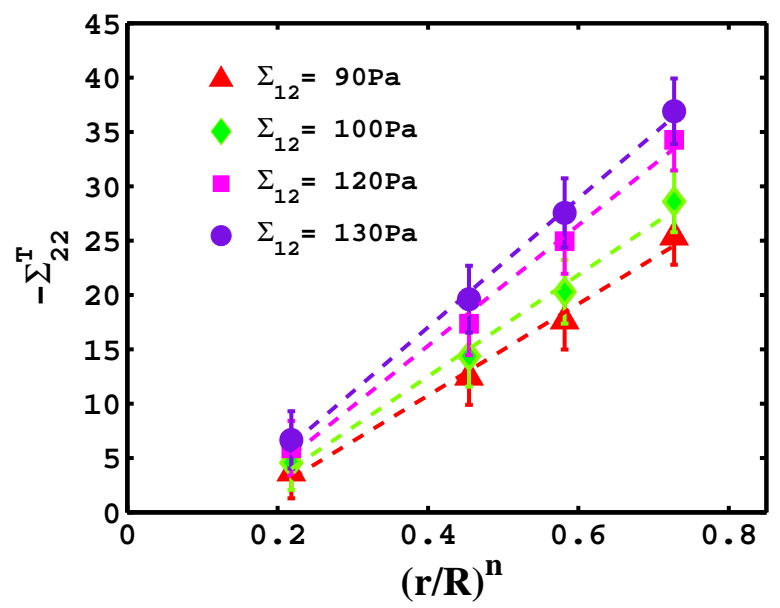

FIG. 5. Axial normal stress, $\Sigma_{22}$, against $(r / R)^{n}$ for a fiber suspension with $n L^{2} d=2.7$ and $a_{r}=10(\phi=0.21)$ and for different values of the shear stress at the rim.

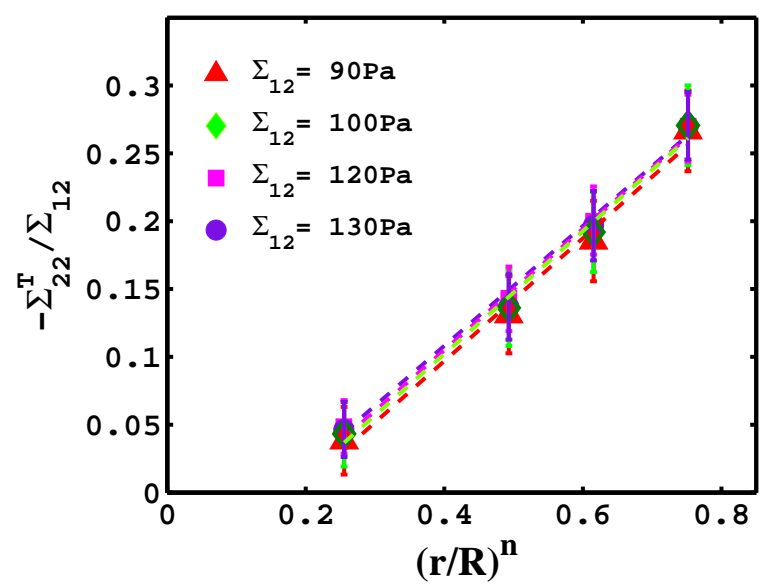

FIG. 6. Axial normal stress normalized by the shear stress at the $\operatorname{rim} \Sigma_{22} / \Sigma_{12}(R)$ against $(r / R)^{n}$ for a fiber suspension with $n L^{2} d=2.7$ and $a_{r}=10(\phi=0.21)$. Within the error bars, all the four normalized profiles collapse, meaning that the normal stress differences are proportional to the shear stress.

is expected on a theoretical basis to occur in a purely Coulombic frictional force scenario where both shear and normal stresses are proportional to the viscous stress. And it is important to verify that it also holds in the shear thinning case where another force scaling arises. From this curve, we calculate the mean slope, A, and the mean origin ordinate, B, from which, according to Eq.(??), we deduce $\alpha_{1}$ and $\alpha_{2}$. The uncertainty in $\mathrm{A}$ and $\mathrm{B}$ is 
evaluated from the slope and the origin ordinate of the straight lines that pass through the extremities of the errorbar data.

\section{RESULTS}

\section{A. Fiber concentration dependence of $\alpha_{1}$ and $\alpha_{2}$}

For three aspect ratios, two fiber lengths and many fiber concentrations, the first and the second normal stress differences $\alpha_{1}$ and $\alpha_{2}$ are extracted from the mean normalized profiles of the axial normal stress that have been obtained for a gap width, $h=2.5 \mathrm{~mm}$. This information is actually important since we will see in the next section that the magnitude of normal stress differences depends on the confinement. The results are given in Fig. ?? and Fig. ?? where $\alpha_{1}$ and $\alpha_{2}$ are plotted against the concentration parameter $n L^{2} d . \alpha_{1}$ is found to be positive and $\alpha_{2}$ is negative. This result $\left(N_{1}>0\right.$ and $\left.N_{2}<0\right)$ has already been obtained by Snook et al. $[?]$. Clearly both $\alpha_{1}$ and $\alpha_{2}$ increase in magnitude when $n L^{2} d$ increases. In addition whatever the length or the aspect ratio of the fibers, $\alpha_{1}$ appears to be almost a function of only $n L^{2} d$, at least for not too high concentrations $\left(n L^{2} d \lesssim 3\right)$, whereas for a given value of $n L^{2} d$, the magnitude of $N_{2}(\phi)$ decreases as the aspect ratio increases.

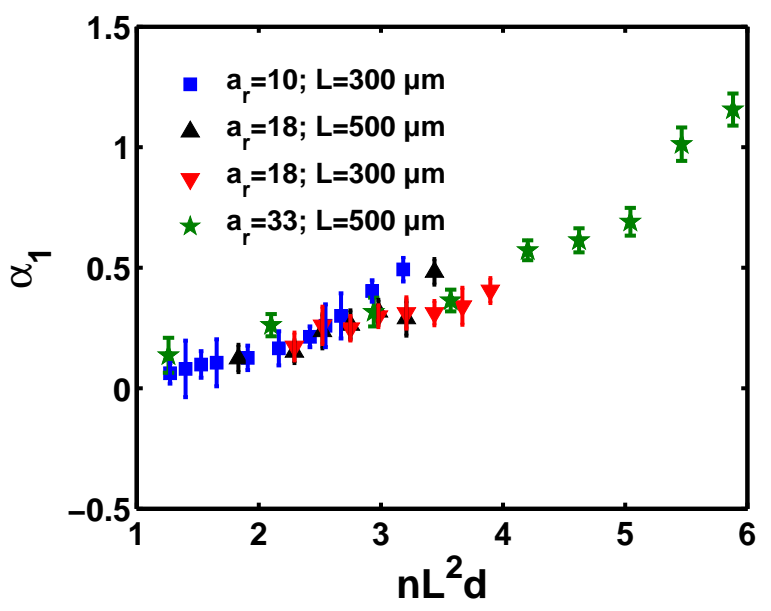

FIG. 7. First normal stress coefficient $\alpha_{1}$ as a function of $n L^{2} d$ as a function of $n L^{2} d$ for three aspect ratios and two fiber lengths.

A quite surprising result is that $\alpha_{2}$ is not negligible compared to $\alpha_{1}$ in contrary to what is often assumed [? ? ]. To highlight this point that will be discussed in detail in Sec. ??, 


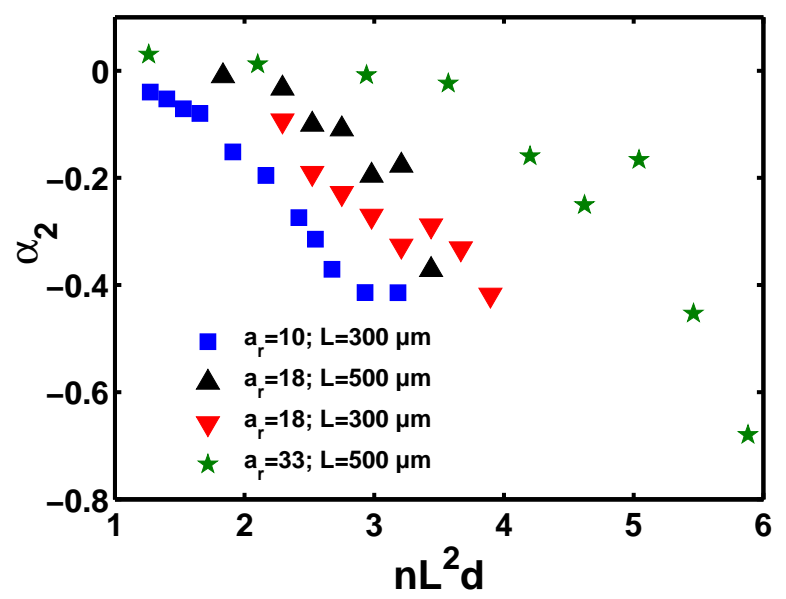

FIG. 8. Second normal stress coefficient $\alpha_{2}$ as a function of $n L^{2} d$ for three aspect ratios and two fiber lengths.

the ratio $-\alpha_{2} / \alpha_{1}$ is plotted against the concentration parameter $n L^{2} d$ in Fig.??. It clearly appears that the ratio $-\alpha_{2} / \alpha_{1}$ increases as the fiber aspect ratio decreases and that $\alpha_{2}$ is much smaller than $\alpha_{1}$ only for high aspect ratios ( $r=33$ in the present study) and low concentrations.

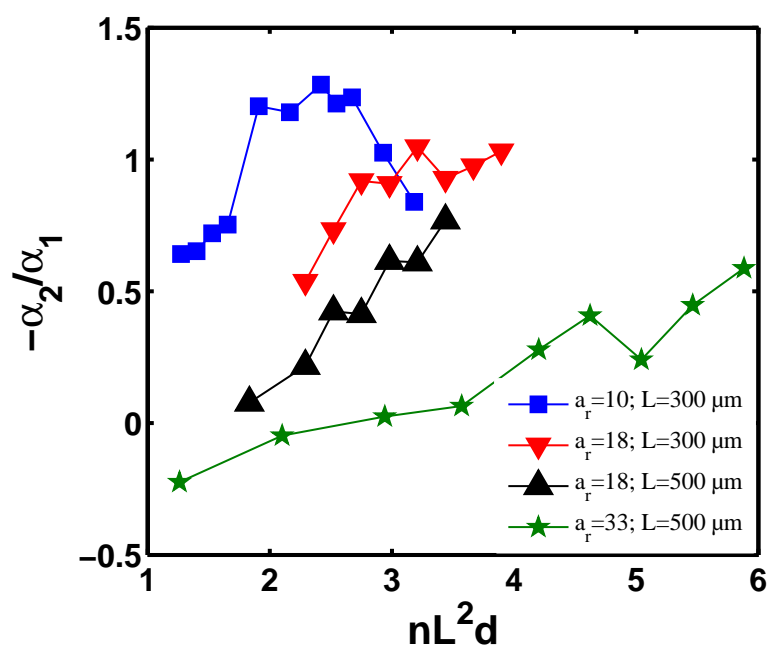

FIG. 9. Ratio $-\alpha_{2} / \alpha_{1}$ as a function of $n L^{2} d$ for 3 aspect ratios and 2 fiber lengths. The gap width was fixed to $2.5 \mathrm{~mm}$. $\alpha_{2}$ is found to be much smaller than $\alpha_{1}$ only for the highest aspect ratio $\left(a_{r}=33\right)$ and the lowest concentrations $\left(n L^{2} d \lesssim 3\right)$ 


\section{B. Effect of the confinement}

We aim to highlight that, as shown in the recent numerical work of Snook [? ], normal stress differences are very sensitive to confinement. Indeed, we carried a systematic study of the effect of the gap width on the resulting values of $\alpha_{1}$ and $\alpha_{2}$. We performed experiments for two fiber aspect ratios, $a_{r}=10$ and $a_{r}=18$, for one fiber length $L=300 \mu m$, and for two fiber concentrations. For each aspect ratio, concentration and gap width, we have determined the shear-thinning index $n$ which reveals that the shear-thinning behaviour of the fiber suspension increases when the confinement parameter, $h / L$, increases. Fig. (??) reports the values of $\alpha_{1}$ and $\alpha_{2}$ for different values of the confinement parameter, $h / L$. It clearly appears that $\alpha_{1}$ increases with confinement (i.e. when h/L decreases). Furthermore, comparing the results obtained for $a_{r}=10$ and $a_{r}=18$ at the same $n L^{2} d$, the effect of confinement on $\alpha_{1}$ is more pronounced for the lower aspect ratio $a_{r}=10$. The same tendency is observed for $\alpha_{2}$, even though it is a little bit less clear. It is surprising that for gap widths as large as $8 \mathrm{~L}$ or $10 \mathrm{~L}$, confinement still plays a role.

\section{DISCUSSION}

Experimental results on normal stress differences available in the literature [? ? ? ? ] mostly deal with relatively low concentrated suspensions (i.e. $n L^{2} d \leq 3$ ). At last, except for Snook et al., all the authors mentioned above performed experiments using parallel plate geometry and obtained the combination $N_{1}-N_{2}$ from the measurement of the total thrust force, $F_{c}$. Fig. ?? displays the variation of $\left(N_{1}-N_{2}\right) / \eta_{f} \dot{\gamma}$ with $n L^{2} d$ that have been obtained by different authors for fibers with aspect ratios of the same order than the ones used in the present study under roughly the same confinement conditions. A first comment on Fig. ?? is that, contrarily to what is presented in [? ], there is a poor agreement between the results of Petrich et al. [? ] and those of Ferec et al. [? ]. Indeed, in the figure 8 of [? ], the normal stresses measured by Petrich et al. [? ] are normalized by $\eta_{f} \dot{\gamma}$ while the results obtained by Ferec et al. [? ] are normalized by $\Sigma_{12}$. Then, when correctly normalized, results displayed in Fig.?? show a significant scatter even for comparable aspect ratios, meaning that new measurements are needed in order to clarify if these large discrepancies come from experimental error or, more likely, from involvement of other parameters such as 

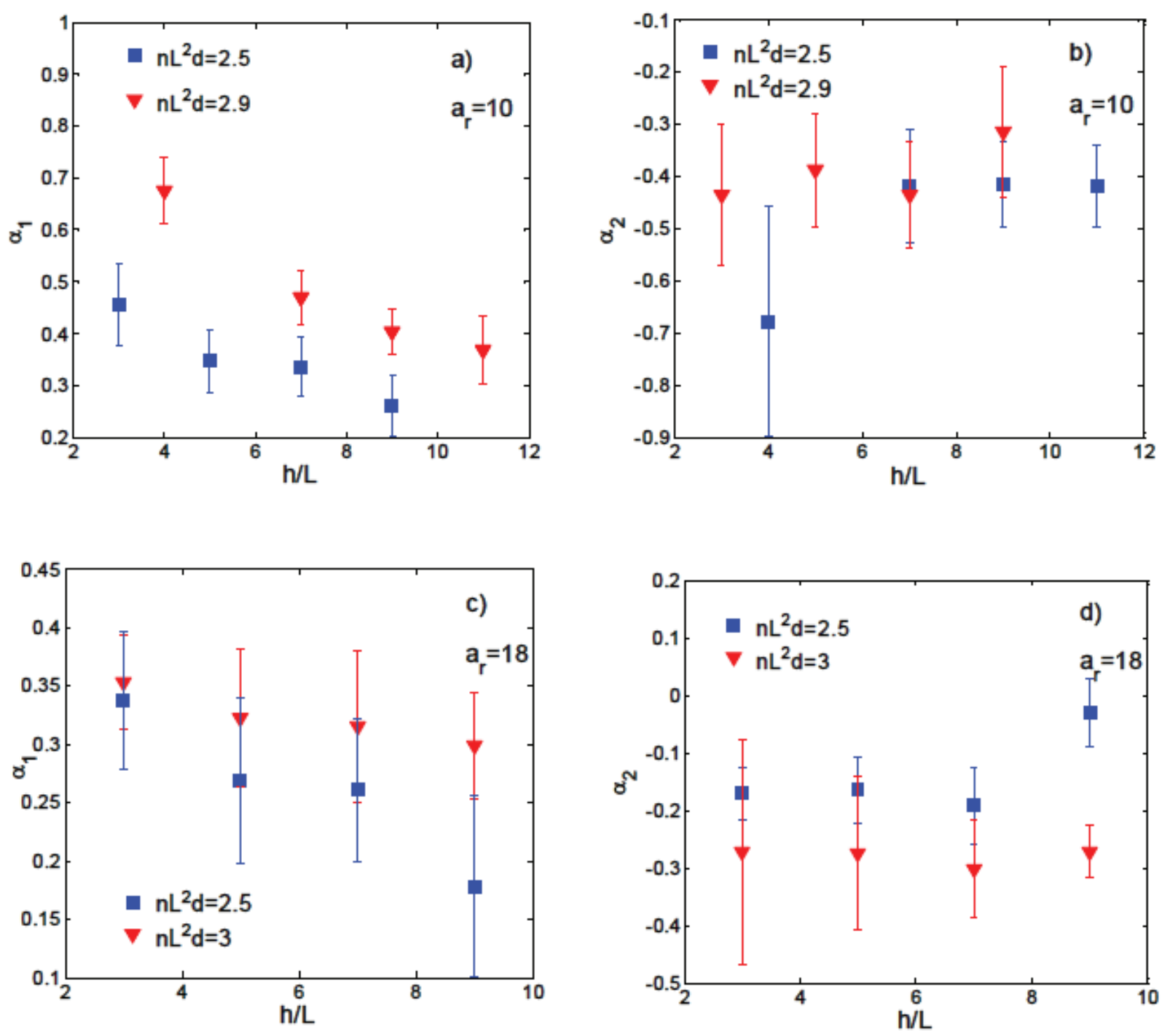

FIG. 10. Effect of the confinement $h / L$ on the magnitude of $\alpha_{1}$ a) and $\alpha_{2}$ b) for $a_{r}=10, n L^{2} d=2.5$ and 2.9. Effect of the confinement $h / L$ on the magnitude of $\alpha_{1}$ c) and $\alpha_{2}$ d) for $a_{r}=18, n L^{2} d=2.5$ and 3

contact forces between fibers that, according to the numerical works of Snook et al. [? ] and Sundararajakumar et al. [? ], are expected to be a key parameter controlling normal stress differences. Note that the results of Snook that are presented here are numerical results obtained using a model which considers hydrodynamic drag forces on each particle and utilises a short-range repulsive force to maintain the excluded volume [? ]. It was not possible to display the associated experimental results [? ] since they have been obtained 
for non confined flows. Indeed, Snook et al. determined the normal stress differences upon measuring the deformation of the free surface of the suspensions in a tilted through and in a Weisenberg rheometer that are both wide gap geometries. The typical dimensions of the flow were 30 to 50 times the fiber length and the authors showed that, in such a non-confined flow, the magnitudes of normal stress differences are much smaller than those expected in a bounded flow. More precisely, Snook compared the values of the normal stress differences obtained from either periodic or confined geometry simulations [? ] and showed that the variation of the gap from $3 \mathrm{~L}$ to $5 \mathrm{~L}$ does not greatly change the values of $N_{1}$ and $N_{2}$ which were, however, quite different from the results obtained with a periodic geometry (infinite gap). Furthermore, the method of free surface flow deformation used by Snook et al. [? ] only gives the ratio of $N_{1}$ and $N_{2}$ to the shear stress (i.e. $\alpha_{1}$ and $\alpha_{2}$ ) while the viscosity of the suspensions has not been measured by the authors. The values of $\alpha_{1}$ and $\alpha_{2}$ measured by Snook et al. are, as expected from the effect of the confinement, much smaller than ours. For instance, to fix ideas, for $\mathrm{r}=32$ and $n L^{2} d=3$, they obtained $\alpha_{2}=0.01 \pm 0.01$ and (as for all aspects ratios and concentrations) $\alpha_{1} \approx-2 \alpha_{2}$ while we measured $\alpha_{1}=-0.33 \pm 0.06$ and $\alpha_{2}=0.08 \pm 0.05$ for $\mathrm{r}=33$.

The numerical work of Snook et al. [? ] also shows that direct mechanical contacts are responsible for an increase of $\alpha_{2}$ and that, in such a case of direct contact between fibers, the magnitude of $N_{2}$ is not much smaller than $N_{1}$ but of the order of $N_{1} / 2$. This is in qualitative agreement with our findings that $\alpha_{2}$ is much smaller than $\alpha_{1}$ only for small values of $n L^{2} d$ and for high aspect ratios ( $a_{r}=33$ in our study). On the opposite, for $a_{r}=10$ and $n L^{2} d>2, N_{2} / N_{1}=O(1)$ (see Fig.??), while the numerical results of Snook [? ] give the same order for the values of the ratio $-\alpha_{2} / \alpha_{1}$, they do not show the same trend, i.e. an increase of $-\alpha_{2} / \alpha_{1}$ when $n L^{2} d$ increases and $a_{r}$ decreases but they rather show an almost constant ratio whatever $a_{r}$ and $n L^{2} d$ (see Table ??).

The experiments of Snook et al. [? ] give a ratio $-\alpha_{2} / \alpha_{1}$ of approximately 0.5 for $n L^{2} d$ comprised between 1.5 and 3 and aspect ratios between 12 and 32 .

Thus, for the ratio $N_{2} / N_{1}$, our results are in qualitative agreement with those of Snook et al. [? ] but the respective values of $\alpha_{1}$ and $\alpha_{2}$ are much larger here than in [? ]. As proposed by Snook et al. [? ] and depicted in Sec. ??, this discrepancy should be explained 


\begin{tabular}{cccccc}
\hline \hline \multicolumn{3}{c}{ Snook (2015) } & & \multicolumn{3}{c}{ Present work } \\
\hline$a_{r}$ & $n L^{2} d=2.5$ & $n L^{2} d=3$ & $a_{r}$ & $n L^{2} d=2.5$ & $n L^{2} d=3$ \\
11 & 0.91 & 0.87 & 10 & 1.2 & 1 \\
20 & 0.78 & 0.87 & 18 & 0.61 & 0.42 \\
30 & 0.80 & 0.75 & 33 & $0.05\left(n L^{2} d=2.1\right)$ & 0.24 \\
\hline \hline
\end{tabular}

TABLE III. Ratio of the magnitude of $\alpha_{2}$ to $\alpha_{1}$ for a confinement 5:1. The ratios obtained in the present work are of the same order as those obtained numerically by Snook [? ]. Nevertheless, while we observe that the ratio $-\alpha_{2} / \alpha_{1}$ increases when $n L^{2} d$ increases and when the aspect ratio decreases, the numerical results of Snook [? ] do not show such trends but display a ratio whose value does almost not depend neither on $n L^{2} d$ nor on $a_{r}$.

by the confinement that is different in Snook's experiments and in ours. Actually, Snook et al. determined the normal stress differences upon measuring the deformation of the free surface of the suspensions in a tilted through and in a Weissenberg rheometer that are both wide gap geometries where the confinement is very weak (the typical dimensions of the geometries are several tens larger than the fiber length). On the opposite, we carried out experiments where the typical confinement is of the order of 5:1 to 8:1. As reported in our experimental study (see Fig. (??)) and shown by the numerical results of Snook [? ], the magnitude of $N_{1}$ and $N_{2}$ increases as the ratio of the gap width to the fiber length decreases. It is worth mentioning that confinement still plays a role for gap with as large as 9 times the fiber length. This point should be kept in mind when measuring normal stress differences which are expected not to depend on characteristic flow dimension only for very wide gaps.

It would have been interesting to further increase the gap in our experiment, in order to check if $\alpha_{1}$ and $\alpha_{2}$ tend to the values obtained by Snook et al. [? ] without confinement. Unfortunately, we were unable to increase the gap width more than $h=11 L$ because the suspension is maintained between the disks by the capillary forces that have to be strong enough to prevent the suspension from flowing out of the gap. On the other hand, it was difficult to explore more confined situations since decreasing the gap increases pressure error due to parallelism defects (see equation (??)). 


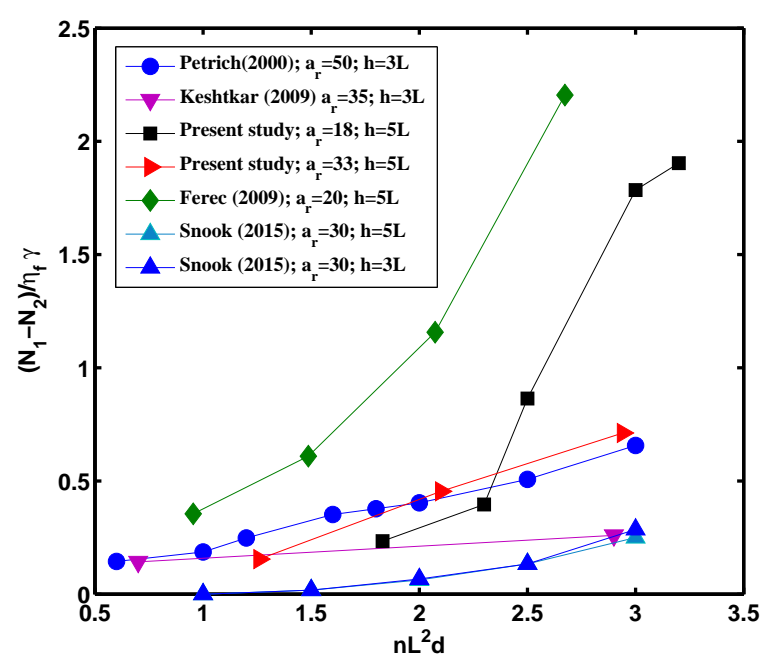

FIG. 11. $N_{1}-N_{2}$ normalized by the shear stress in the fluid, $\eta_{f} \dot{\gamma}$ versus $n L^{2} d$. Comparison of our results with those obtained from the measurement of the thrust force in rotating parallel plate geometry [? ? ? ] and with the numerical results of Snook [? ].

\section{CONCLUSION}

In this work we have presented an experimental method to determine the two normal stress differences $N_{1}$ and $N_{2}$ independently for suspensions of non-Brownian fibers dispersed in a Newtonian fluid. Both the first and second normal stress differences have been measured for a large range of aspect ratios and fiber concentrations so that semi-dilute and concentrated regimes have been explored. The results show that $N_{1}$ is positive and $N_{2}$ negative, and both increase in magnitude with $n L^{2} d$. Unlike what is often assumed in the literature, we measure a non zero value of $N_{2}$ and $\alpha_{2}$ is negligible with respect to $\alpha_{1}$ only for large aspect ratios and low fiber concentrations. For aspect ratios smaller than or equal to 20, $\alpha_{1}$ and $\alpha_{2}$ are found to be of the same order of magnitude. This is in qualitative agreement with the experimental results of Snook et al. [? ]. Nevertheless, we measured much more larger values of $\alpha_{1}$ and $\alpha_{2}$ than these authors. We show that confinement can explain these differences since $\alpha_{1}$ and $\alpha_{2}$ measurements depend on the ratio of the gap width to the fiber length even for ratios as large as 8 or 10. The comparison of our results with most previous measurements of $N_{1}-N_{2}$ obtained from the thrust force exerted on the rotating disks in a torsionnal flow is rather good. 


\section{ACKNOWLEDGMENTS}

The authors are grateful to Région Provence Alpes Côte d'Azur for funding one of us, to La société nouvelle de flockage for supplying the fibers and to G. Sauder, F. Juan, F. Lippi, C. Pitiot and J.L. Vanni for their technical support.

\section{Appendix: Effect of fiber surface properties on normal stress differences}

In this appendix, we would ike to present very preliminary results on the effect of fiber surface properties on the normal stress differences. Indeed, most theoretical or numerical studies have focused on the role played by the hydrodynamic interactions (long range or lubrication forces) between fibers in the rheological behaviour of fiber suspensions but, a few studies [? ? ? ] have shown that direct mechanical contacts between fibers play a crucial role in fiber suspension rheology. In particular, direct contacts are responsible for an increase of the frequency of rotation of fibers in the shear flow as $n L^{2} d$ becomes $\mathrm{O}(1)$ that, in turn, increases both the viscosity and the normal stress differences. To verify experimentally these numerical results it would be worth modifying the solid contacts between fibers. A possible way to do this is to modify the fiber surface. Since the fibers we used are flock fibers, they have been subjected to a finishing process and are coated. Even though very little is known about the process they underwent, according to the information given by the manufacturer, washing the fibers before adding them to the suspending liquid should modify their surface properties and, subsequently their mechanical contacts.

We have conducted experimental measurements of $\alpha_{1}$ and $\alpha_{2}$ for four fiber concentrations, $n L^{2} d=4.24 .6,5.5$ and $5.9(\phi=0.10,0.11,0.13$ and 0.14$)$ for the highest aspect ratio, $a_{r}=33$. In Fig. ?? and ??, we report the comparison of the results of $\alpha_{1}$ and $\alpha_{2}$ obtained either with washed or unwashed fibers. The magnitude of $\alpha_{1}$ is higher for the washed fibers than for the unwashed ones and the difference increases as $n L^{2} d$ increases. On the contrary, within the error bars, $\alpha_{2}$ is the same for washed or unwashed fiber suspensions even though 


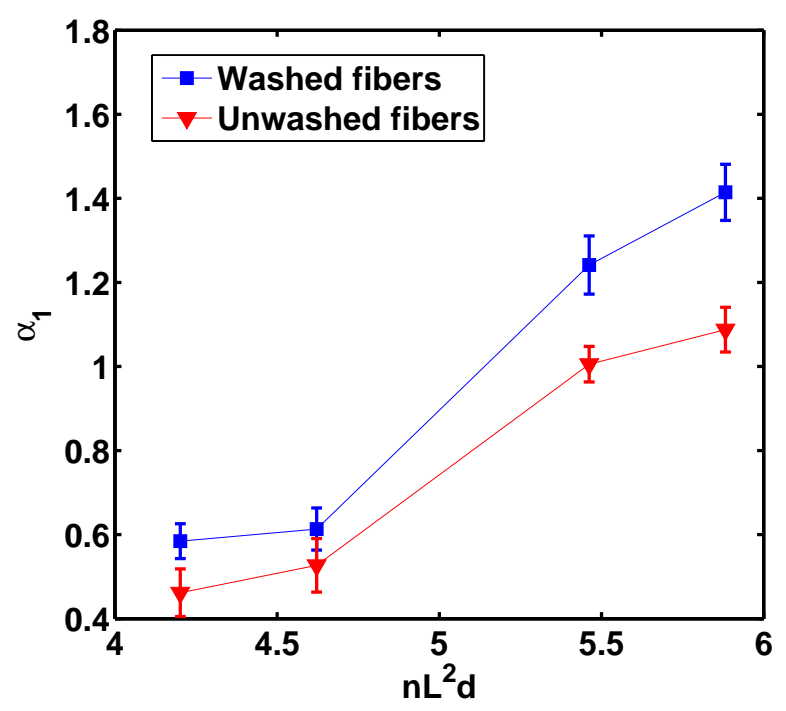

FIG. 12. First normal stress coefficients, for $a_{r}=33$ measured for washed (blue squares) or unwashed (red triangles) fibers.

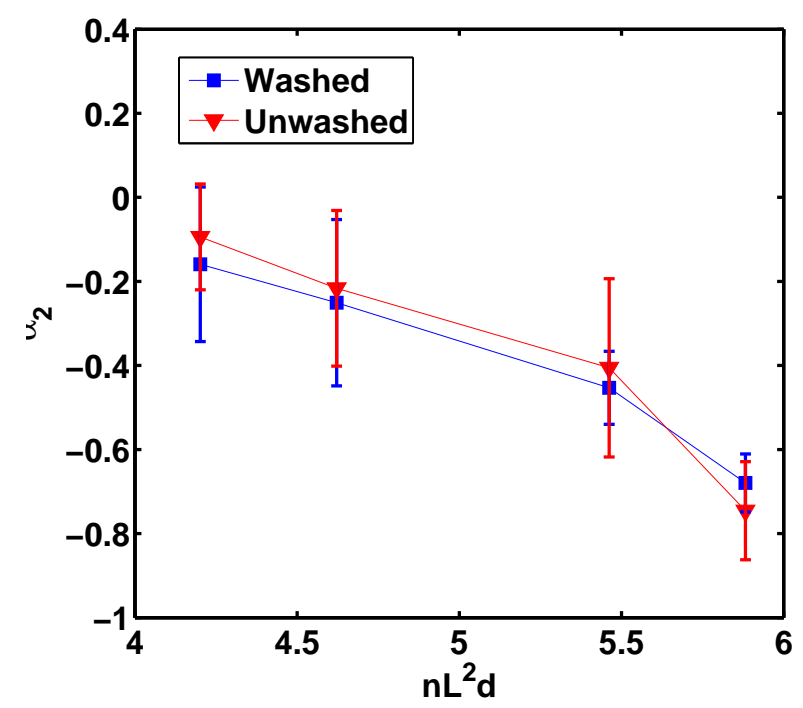

FIG. 13. Second normal stress coefficients, for $a_{r}=33$ measured for washed (blue squares) or unwashed (red triangles) fibers.

the magnitude of $\alpha_{2}$ is always slightly higher for washed than for unwashed fibers.

To interpret in a conclusive way the increase of the magnitude of the normal stress differences by enhanced contact forces, it would be worth measuring fiber-fiber interaction in both cases of washed and unwashed fibers. Petrich et al. [? ] and Chaouche et al. [? 
] proposed experimental methods to measured both normal and frictional forces between contacting fibers. These methods should be used to explore quantitatively the relationship between contact interactions and normal stress differences. 
[] batchelor1971stressBatchelor, G. 1971. The stress generated in a non-dilute suspension of elongated particles by pure straining motion The stress generated in a non-dilute suspension of elongated particles by pure straining motion. Journal of Fluid Mechanics4604813-829.

[] bird1977dynamicsBird, RB., Armstrong, RC., Hassager, O. Curtiss, CF. 1977. Dynamics of polymeric liquids Dynamics of polymeric liquids (1). Wiley New York.

[] boyer2011denseBoyer, F., Pouliquen, O. Guazzelli, É. 2011. Dense suspensions in rotatingrod flows: normal stresses and particle migration Dense suspensions in rotating-rod flows: normal stresses and particle migration. Journal of Fluid Mechanics6865-25.

[] brenner1974rheologyBrenner, H. 1974. Rheology of a dilute suspension of axisymmetric Brownian particles Rheology of a dilute suspension of axisymmetric brownian particles. International journal of multiphase flow12195-341.

[] CarterCarter, LF. 1967. A Study of the Rheology of Suspensions of Rod-Shaped Particles in a NavierStokes Liquid A Study of the Rheology of Suspensions of Rod-Shaped Particles in a NavierStokes Liquid Ph.D. Thesis. University of Michigan, Ann Arbor.

[] chaouche2001rheologyChaouche, M. Koch, DL. 2001. Rheology of non-Brownian rigid fiber suspensions with adhesive contacts Rheology of non-brownian rigid fiber suspensions with adhesive contacts. Journal of Rheology452369-382.

[] couturier2011suspensionsCouturier, É., Boyer, F., Pouliquen, O. Guazzelli, É. 2011. Suspensions in a tilted trough: second normal stress difference Suspensions in a tilted trough: second normal stress difference. Journal of Fluid Mechanics68626-39.

[] dbouk:tel-00673964Dbouk, T. 2011. Rheology of concentrated suspensions and shear-induced migration Rheology of concentrated suspensions and shear-induced migration ThesesUniversité Nice Sophia Antipolis. https://tel.archives-ouvertes.fr/tel-00673964

[] dbouk2013normalDbouk, T., Lobry, L. Lemaire, E. 2013. Normal stresses in concentrated non-Brownian suspensions Normal stresses in concentrated non-brownian suspensions. Journal of Fluid Mechanics715239-272.

[] djalili2005modelDjalili-Moghaddam, M. Toll, S. 2005. A model for short-range interactions in fibre suspensions A model for short-range interactions in fibre suspensions. Journal of non-newtonian fluid mechanics132173-83. 
[] doi1988theoryDoi, M. Edwards, SF. 1988. The theory of polymer dynamics The theory of polymer dynamics ( 73). oxford university press.

[] fan1998directFan, X., Phan-Thien, N. Zheng, R. 1998. A direct simulation of fibre suspensions A direct simulation of fibre suspensions. Journal of Non-Newtonian Fluid Mechanics741113135.

[] ferec2009modelingFérec, J., Ausias, G., Heuzey, M. Carreau, P. 2009. Modeling fiber interactions in semiconcentrated fiber suspensions Modeling fiber interactions in semiconcentrated fiber suspensions. Journal of Rheology53149-72.

[] folgar1984orientationFolgar, F. Tucker, CL. 1984. Orientation behavior of fibers in concentrated suspensions Orientation behavior of fibers in concentrated suspensions. Journal of Reinforced Plastics and Composites3298-119.

[] forgacs1959particleForgacs, O. Mason, S. 1959. Particle motions in sheared suspensions: IX. Spin and deformation of threadlike particles Particle motions in sheared suspensions: Ix. spin and deformation of threadlike particles. Journal of colloid science145457-472.

[] gamonpilas2016shearGamonpilas, C., Morris, JF. Denn, MM. 2016. Shear and normal stress measurements in non-Brownian monodisperse and bidisperse suspensions Shear and normal stress measurements in non-brownian monodisperse and bidisperse suspensions. Journal of Rheology602289-296.

[] ganani1985suspensionsGanani, E. Powell, R. 1985. Suspensions of rodlike particles: literature review and data correlations Suspensions of rodlike particles: literature review and data correlations. Journal of composite materials193194-215.

[] goto1986flowGoto, S., Nagazono, H. Kato, H. 1986. The flow behavior of fiber suspensions in Newtonian fluids and polymer solutions. The flow behavior of fiber suspensions in newtonian fluids and polymer solutions. Rheologica acta253246-256.

[] jeffery1922motionJeffery, GB. 1922. The motion of ellipsoidal particles immersed in a viscous fluid The motion of ellipsoidal particles immersed in a viscous fluid. Proceedings of the Royal Society of London. Series A102715161-179.

[] keentok1999edgeKeentok, M. Xue, SC. 1999. Edge fracture in cone-plate and parallel plate flows Edge fracture in cone-plate and parallel plate flows. Rheologica acta384321-348.

[] keshtkar2009rheologicalKeshtkar, M., Heuzey, M. Carreau, P. 2009. Rheological behavior of fiber-filled model suspensions: effect of fiber flexibility Rheological behavior of fiber-filled 
model suspensions: effect of fiber flexibility. Journal of Rheology533631-650.

[] lindstrom2009numericalLindström, SB. Uesaka, T. 2009. A numerical investigation of the rheology of sheared fiber suspensions A numerical investigation of the rheology of sheared fiber suspensions. Physics of Fluids218083301.

[] mackaplow1996numericalMackaplow, MB. Shaqfeh, ES. 1996. A numerical study of the rheological properties of suspensions of rigid, non-Brownian fibres A numerical study of the rheological properties of suspensions of rigid, non-brownian fibres. Journal of Fluid Mechanics329155-186.

[] pressureinetrtMcCoy, D. Denn, M. 1971. Secondary flow in a parallel-disk viscometer Secondary flow in a parallel-disk viscometer. Rheologica Acta103408-411.

[] mongruel1999shearMongruel, A. Cloitre, M. 1999. Shear viscosity of suspensions of aligned non-Brownian fibres Shear viscosity of suspensions of aligned non-brownian fibres. Rheologica $\operatorname{acta} 385451-457$.

[] natale2014rheologicalNatale, G., Heuzey, M., Carreau, P., Ausias, G. Férec, J. 2014. Rheological modeling of carbon nanotube suspensions with rod-rod interactions Rheological modeling of carbon nanotube suspensions with rod-rod interactions. AIChE Journal6041476-1487.

[] petrich1998interactionsPetrich, MP. Koch, DL. 1998. Interactions between contacting fibers Interactions between contacting fibers. Physics of Fluids1082111-2113.

[] petrich2000experimentalPetrich, MP., Koch, DL. Cohen, C. 2000. An experimental determination of the stress-microstructure relationship in semi-concentrated fiber suspensions An experimental determination of the stress-microstructure relationship in semi-concentrated fiber suspensions. Journal of non-newtonian fluid mechanics952101-133.

[] schmid2000simulationsSchmid, CF., Switzer, LH. Klingenberg, DJ. 2000. Simulations of fiber flocculation: Effects of fiber properties and interfiber friction Simulations of fiber flocculation: Effects of fiber properties and interfiber friction. Journal of Rheology (1978-present)444781809.

[] sepehr2004rheologicalSepehr, M., Carreau, PJ., Moan, M. Ausias, G. 2004. Rheological properties of short fiber model suspensions Rheological properties of short fiber model suspensions. Journal of Rheology4851023-1048.

[] shaqfeh1990hydrodynamicShaqfeh, ES. Fredrickson, GH. 1990. The hydrodynamic stress in a suspension of rods The hydrodynamic stress in a suspension of rods. Physics of Fluids A: 
Fluid Dynamics217-24.

[] snookPHDSnook, B. 2015. The dynamics of the microstructure and the rheology in suspensions of rigid particles The dynamics of the microstructure and the rheology in suspensions of rigid particles . University of Florida.

[] snook2014normalSnook, B., Davidson, LM., Butler, JE., Pouliquen, O. Guazzelli, E. 2014. Normal stress differences in suspensions of rigid fibres Normal stress differences in suspensions of rigid fibres. Journal of Fluid Mechanics758486-507.

[] sundararajakumar1997structureSundararajakumar, R. Koch, DL. 1997. Structure and properties of sheared fiber suspensions with mechanical contacts Structure and properties of sheared fiber suspensions with mechanical contacts. Journal of Non-Newtonian Fluid Mechanics733205-239.

[] zarraga2000characterizationZarraga, IE., Hill, DA. Leighton Jr, DT. 2000. The characterization of the total stress of concentrated suspensions of noncolloidal spheres in Newtonian fluids The characterization of the total stress of concentrated suspensions of noncolloidal spheres in newtonian fluids. Journal of Rheology442185-220.

[] zirnsak1994normalZirnsak, M., Hur, D. Boger, D. 1994. Normal stresses in fibre suspensions Normal stresses in fibre suspensions. Journal of non-newtonian fluid mechanics54153-193. 